\title{
Shatảpeare'
}

\section{Dramatifde $\mathfrak{a} \mathfrak{a} \mathfrak{e} \mathfrak{e}$.}

ueberfegt

oon

2(uguft $\mathfrak{S}$ Bilffelm von Sdjlegel,

ergángt und erláutert

on

Rubmig Siect.

\section{Teunter Theil.}

бimberine.

siebes sido und suft.

Diomeo und Julia.

Miacbetr),

$$
\begin{aligned}
& \text { Berlin, } \\
& \text { b y G. Mcimcr. } \\
& 1833 \text {. }
\end{aligned}
$$


